CARNETS DE Carnets de géographes

GÉOGRAPHES.

5 | 2013

Géographie humanimale

\title{
Les relations humains/animaux
}

De l'espace protégé à l'espace partagé, une géographie physique et sensible

Stephanie Chanvallon

\section{(2) OpenEdition}

Journals

Édition électronique

URL : http://journals.openedition.org/cdg/1057

DOI : $10.4000 /$ cdg. 1057

ISSN : 2107-7266

Éditeur

UMR 245 - CESSMA

Référence électronique

Stephanie Chanvallon, "Les relations humains/animaux », Carnets de géographes [En ligne], 5 | 2013, mis en ligne le 01 janvier 2013, consulté le 21 décembre 2020. URL : http://journals.openedition.org/ cdg/1057 ; DOI : https://doi.org/10.4000/cdg.1057

\section{(c) $\Theta \Theta \Theta$}

La revue Carnets de géographes est mise à disposition selon les termes de la Licence Creative Commons Attribution - Pas d'Utilisation Commerciale - Pas de Modification 4.0 International. 


\title{
Les relations humains/animaux ${ }^{1}$ \\ De l'espace protégé à l'espace partagé, une géographie physique et sensible
}

\author{
STEPHANIE CHANVALLON \\ Anthropologue, Chercheur associé, \\ Laboratoire Violences, Identités, Politiques et Sports (VIPS), \\ Université européenne de Bretagne \\ stephanie-chanvallon@netcourrier.com
}

\section{Résumé}

Les relations de l'Homme à l'animal sont appréhendées avec la notion d'espace : espace vital comme distance de sécurité, espace de proximité sous contrôles, jusqu'à l'espace intime et privilégié lors de rencontres parfois extraordinaires. Si dans certaines conditions l'espace est défini par avance, structuré et limitatif, il peut être en perpétuelle construction et évolution en fonction des représentations et intentions d'agir, en fonction aussi de cet autre animal qui fuit ou se rapproche, qui décide ou non de rencontrer l'humain. Quand l'espace partagé entre ces deux espèces s'est établi sur une communication et une confiance réciproque, il acquiert une autre dimension, celle d'un " entre-deux animal » dont l'agencement unique témoigne des potentialités relationnelles.

\begin{abstract}
Man's relationship with animal are apprehended with the notion of space: space as a vital safety distance, space proximity under controls, to the intimate and exclusive space during sometimes extraordinary meetings. So under certain conditions space is defined in advance, structured and restrictive, it may be in perpetual construction and development according to the representations and intentions to act, according also to this other animal which flees or approaches, which or not decides not meet the human. When the space shared between these two species is established on a communication and a mutual trust, it acquires another dimension, that of an "animal interval" whose single fitting reflects the relational potentialities.
\end{abstract}

\footnotetext{
${ }^{1}$ Par souci de simplification, j'emploie ici le terme " animaux » pour parler des « animaux non humains ", vocable largement utilisé aujourd'hui dans la littérature scientifique. Le terme « Homme » désigne par ailleurs l'être humain au sens large, hommes et femmes confondus.
} 
Les animaux font parler d'eux alors qu'ils sont nombreux à disparaître de leurs espaces naturels. En France, ils reconquièrent des territoires et les voici arrivés dans la Cité. Au sens propre par leur présence croissante aux abords et au cœur des villes et au sens figuré au travers des différentes problématiques scientifiques, tout particulièrement en sciences humaines. Le statut de l'animal évolue et encourage la mise en place d'études sociologiques sur la puissance des interactions Homme/Animal. Les expériences personnelles de rencontres et de côtoiements d'animaux sauvages ouvrent un espace de perception, qu'il soit géographique ou psychique. Observer, s'imprégner de la nature, rencontrer l'animal, c'est déjà le découvrir vraiment et l'apprendre, accepter de le voir autrement et se laisser surprendre. Mais si nous pouvons écrire sur les animaux, nous ne pourrons jamais exprimer à leur place. Quoi qu'il en soit, nous sommes spectateurs et toujours participants de cet « immense chantier vivant où forme et territoire s'entrecroisent et proposent à chaque espèce et à chaque individu la pente de sa signature » (Bailly, 2007 : 96).

Pour répondre à ce numéro spécial des Carnets de géographes cet article s'appuie sur des recherches en anthropologie sociale et pluridisciplinaires analysant spécifiquement les relations de l'Homme à la Nature et à l'Animal sauvage, menées à partir " d'entretiens au long cours » (Chanvallon, 2009). Le texte sera ainsi illustré de propos d'enquêtés (en italique dans le texte) et du vécu de l'auteur ${ }^{2}$. Les relations anthropozoologiques seront abordées sous l'angle spatial. A partir d'expériences de terrain, l'espace géographique, territoire propre à chaque être vivant et lieu des rencontres Homme/Animal, est exposé dans l'ordre successif des espaces protégés aux espaces partagés, entre distance de fuite et contacts physiques. Différentes situations viendront témoigner de comportements atypiques qui interpellent. En derniers lieux cet article présentera ce que nous appelons l'« entredeux animal», puis une approche de l'espace comme zone de rencontre motivée par le désir " d'être en relation ".

Nous focaliserons essentiellement sur les relations avec les animaux sauvages ${ }^{3}$. "Sauvage " sera employé à propos des animaux qui vivent sur leur territoire naturel et qui décident ou non de rencontrer l'espèce humaine. II ne faut pas voir ici une vision idéalisée et idyllique de l'animal ni de la rencontre entre un être humain et un animal - potentiellement dangereuse. Dans ces mondes animaux, il y a des temps pour s'alimenter, se reproduire, se protéger, se reposer, jouer, et puis il y a des interstices ouverts pour la curiosité (Lorenz, 1985). Nombre d'espèces témoignent d'un intérêt pour l'espèce humaine, se rapprochent et créent des interfaces. "Je pense qu'ils cherchent aussi à interagir avec nous. Les orques invitent les hommes ", souligne Marc, plongeur apnéiste d'une quarantaine d'années, organisateur de croisières à la rencontre des orques dans les eaux de Norvège.

\footnotetext{
${ }^{2}$ Peut-être est-ce un avantage en tant qu'anthropologue du social qui explore ce domaine d'être soi-même acteur dans une telle aventure pour en entrevoir tant l'intérêt, la richesse que la difficulté à évoluer entre objectivité et subjectivité, implication et mise à distance de son sujet d'étude.

${ }^{3}$ Mais qu'est-ce qu'un animal sauvage ? Est-ce un animal qui n'a jamais côtoyé l'espèce humaine ? Comment qualifier alors ces dauphins solitaires qui viennent partager du temps avec l'être humain ?
} 


\section{Une méthodologie entre sciences et expériences}

\section{Une anthropologie sociale qualitative et réflexive}

Les enquêtés sont des professionnels et des passionnés de nature choisis parce qu'ils étaient susceptibles de " fournir les informations importantes qui ne [pouvaient] pas être aussi bien obtenues en suivant d'autres choix » (Maxwell, 1999 : 128). Ils sont par exemple biologiste, cinéaste, ethnologue, navigateur professionnel, plongeur apnéiste. Vingt-deux récits de vie ont été analysés dans une approche sensible et réflexive (Céfaï, 2003), valorisant ainsi la part de singularité de ces femmes et de ces hommes (Cuche, 2004 ; Héas, 2010), leurs " stratégies identitaires ". Dans cette perspective, les mots et leurs résonances, notamment affectives, importent plus que le nombre d'entretiens ou la fréquence des vocables utilisés. Ce qui est recherché dans leurs témoignages est l'expression de leur vécu, ce qui fait sens pour eux dans leurs relations privilégiés avec la nature et l'autre animal, initie une autre "façon » de les regarder (Gould, 2001) et amène un retour bénéfique sur soi (Ormiston, 2003). Les subjectivités et l'implication du chercheur auprès de ces informateurs privilégiés et sur le terrain agissent ici comme " levier et instrument de connaissance " (Lavigne, 2007). Pour Le Grand (2006), il s'agit de reconnaître dans l'engagement du chercheur et ses propres expériences une " potentialité heuristique ».

\section{A propos des anecdotes}

A propos des animaux, "The "anecdotal method" is gaining momentum in animal behaviour " (Bates \& Byrne, 2007). Pour que les anecdotes soient utilisées comme données scientifiques, ces auteurs posent plusieurs conditions ${ }^{4}$. Mais la qualité d'une anecdote ne peut être sous-estimée parce que rapportée par un informateur privilégié et non par le chercheur lui-même, d'où l'investissement réalisé dans " les entretiens au long cours » et la compréhension de l'agencement des expériences parfois extraordinaires. Un comportement anecdotique, aussi singulier soit-il, est un témoignage privilégié d'un vaste réservoir de possibles (Gould, 1997). "L'utilisation d'anecdotes nous permet d'appréhender l'étendue des comportements des espèces qui se déroulent en milieu naturel, leurs aptitudes les plus créatives, plutôt que de nous restreindre aux paradigmes déjà répandus sur l'étude des autres espèces animales et humaines $\nu^{5}$ (Bates \& Byrne, 2007). II semble judicieux que le chercheur qui relève l'anecdote ou l'enquêté qui en témoigne aient des connaissances sur l'espèce observée mais à condition qu'elles ne soient un frein en termes de comportements attendus. Comme le souligne Cardonne, "C'est le milieu qui détermine son occupant. Car, cela est certain, beaucoup de changements interviendront d'une population à une autre même si la latitude et les conditions nous paraissent identiques » (2004 : 109).

\footnotetext{
${ }^{4}$ L'observateur doit être expérimenté, il "sait » de quoi il parle, il connaît l'espèce dont il a observé un comportement anecdotique. - Pour se préserver de l'anthropomorphisme et de tout autre mésinterprétation, il faut s'en tenir à ce qui est observé. - Sont utilisées des versions initiales des descriptions (non transformées avec le temps) qui ont elles-mêmes été réalisées juste après l'événement.

${ }^{5}$ Traduction personnelle : "Using anecdotal records allow us to consider the full range of behaviour that occurs naturally in a species, including their most creative aptitudes, rather than restricting the focus to paradigms already familiar from systematic study of other animal species and humans".
} 


\section{"Chacun chez soi »}

Le territoire est un espace vital, lieu de nourriture, de protection, de séduction, de reproduction, de socialisation, un espace où se jouent de multiples relations avec ceux de la même espèce ou d'une espèce différente. Les limites du territoire, ces zones à ne pas franchir lorsqu'on est un intrus, une menace potentielle, sont signifiées par des marques, des odeurs, par le comportement de l'animal qui peut attaquer ou prendre la fuite (Hall, $1966^{6}$ ). Dans les rencontres fortuites entre des êtres humains et des animaux sauvages, en dehors des comportements de prédation, les attaques sont souvent liées à notre incapacité à identifier ou comprendre la situation et les signes en présence : nous avons pénétré dans un espace où nous n'étions pas invités. Pourtant, bien des espèces adoptent des attitudes qui peuvent nous interpeller, que ce soit les rapaces, les chiens de troupeaux, les ours, les requins, etc.

Nous ne partageons pas avec les animaux le même mode d'expression. L'absence de compréhension est d'ailleurs réciproque, or nous avons tendance à ne voir que leur manque : ils sont dénués de parole. Mais depuis leur place, la situation est similaire : nous ne pouvons nous faire comprendre. Cependant, il existe un lieu commun dans les cris, les postures particulières, les mouvements du corps et les rythmes, les vibrations peut-être. La communication non-verbale (Hennel-Brzozowska, 2008) - déjà décrite par Darwin en 1872 est ainsi un mode d'échange que les humains peuvent avoir avec les animaux. L'animal qui n'est pas sur son territoire où s'y sent menacé, a une distance de fuite ou de sécurité, celle subtile dont nous pouvons faire l'expérience quotidiennement dans nos relations avec autrui (de l'espace proche à l'espace intime). Il faut pouvoir la respecter et ne pas enfreindre cette règle naturelle. Souvent, l'être humain montre son ignorance, son incompréhension. Nous sommes tellement habitués à vivre dans des espaces supra-codés, qu'il semble que nous nous comportions comme si les espaces sauvages étaient vierges de signes, dénués de sens, tels des "taupes monomaniaques" (Picq, 2005). La transgression des limites est parfois volontaire. L'empiètement sur le territoire de l'autre, l'espace qu'il s'est approprié, peut déclencher un combat. Perdu par le "tenant du titre ", une redéfinition du territoire se fera en faveur du " vainqueur ». Parfois, l'être humain provoque et met l'autre au défi. Il sait qu'il n'est pas chez lui mais il veut affronter l'animal sur son territoire. Inconscience, volonté de puissance ou jeu instinctif ? La distance de sécurité se transforme alors en distance critique qui peut mener à l'attaque-défense.

"Au cours d'une plongée en Mer de Cortez, j'avais remarqué qu'un groupe de femelles otaries était rassemblé au loin. Je continuais mon exploration sous-marine tout en gardant mes distances. Le matin même, un mâle de taille conséquente était venu vers moi en poussant des cris mais sans agressivité : je ne devais pas m'approcher davantage. J'étais donc à l'écart, et quelle ne fut pas ma surprise quand une otarie - sans nul doute très curieuse vint vers moi, progressivement, par une approche prudente et douce. Au bout de quelques minutes, nous étions là face à face, tranquilles, jouant à nous imiter l'une l'autre. Soudain, j'ai entendu les cris du mâle : il venait chercher la femelle qui avait quitté le " harem ». Par deux fois elle a tenté une nouvelle escapade pour me rejoindre et par deux fois le mâle est venu la

\footnotetext{
${ }^{6}$ Cité in Henri Galinié (07/02/01), « Utiliser la notion de « distance critique » dans l'étude de relations sociospatiales ", Les petits cahiers d'Anatole, $\mathrm{n}^{\circ} 7$.
} 
chercher. N'étant pas sur leur territoire, je ne me suis pas sentie menacée, ce n'était pas moi la rebelle, je n'avais pas à m'éloigner. »

Le " chacun chez soi » est une limite donnée pour assurer l'intégrité physique et psychique. Mais la frontière entre l'individu et le monde est mouvante. Comme le précise Serres : " La première [limite], intérieure, protège l'habitant de sa douceur; à l'extérieur, la dernière menace, de ses duretés, les envahisseurs possibles. En celle du milieu se percent des pores, des passages, portes ou porosités par lesquelles, et souvent par semi-conduction, tel vivant ou telle chose entre, se verrouille, sort, transite, attaque, attend sans espoir... (...) Défendre, protéger, interdire ou laisser passer : ainsi, triplement, fonctionne une frontière " (2008: 45). Ces frontières peuvent être poreuses et ouvrir à des voies d'échanges où les espaces sont confondus pour en créer un nouveau.

Pour préserver les animaux sauvages menacés de disparition, il semble aujourd'hui important d'éviter toute mise en présence (Bériot, 2012). Toute rencontre peut en effet développer une sorte de familiarité et réduire l'espace de sécurité de l'animal par habituation à la présence humaine. La pratique du braconnage en devient ainsi facilitée. Pour exemple, les tigres sont chassés de leur territoire et amenés à vivre sur des espaces de plus ou en plus réduits, ou bien ils sont exterminés et même importés dans des espaces clos: des élevages pour alimenter un commerce alimentaire, aphrodisiaque et ornemental (Bériot, 2012). Du sauvage au domestique d'élevage, il n'y a qu'un pas: la violation des frontières du vivant et le non respect de la vie dans sa dimension même symbolique et sacrée. Ne pas habiter des espaces naturels pour protéger des espèces, c'est aussi accepter de ne pas céder à la tentation d'être les privilégiés de telles rencontres sous couvert d'un éco-tourisme.

\section{Chez eux et pourtant nuisibles}

L'espace naturel d'un animal sauvage est parfois incompatible avec les activités humaines. A côté d'une nomenclature biologique pour la classification du vivant, une liste définit les espèces dites emblématiques, celles à protéger (Liste Rouge internationale), celles sans valeur patrimoniale, animaux de laboratoire, etc. Pour les espèces dites nuisibles, il s'agit dans la majorité des cas d'animaux qui vivent sur leur territoire naturel et que nous partageons avec eux : renards, fouines, geai des chênes, etc. Ils ne sont nuisibles que par rapport à nos pratiques et bien souvent il est question d'un conflit de territoire décrété par l'Homme : ces animaux des champs et des plaines ont les mêmes proies que celle des chasseurs, ils leur font concurrence et se nourrissent de surcroît de leur gibier élevé à grand frais. Pourtant, pour le bon déroulement des affaires de la chasse, des animaux à l'origine sauvage: faisans, perdrix, sont élevés dans des espaces évidemment non sauvages, pour être ensuite relâchés dans leur milieu naturel. Mais imprégnés par l'Homme, nombre d'entre eux ont perdu leurs instincts, en l'occurrence la fuite ou le camouflage.

"Je me souviens de cette perdrix - une rescapée de la chasse amputée d'une patte - et tout juste recueillie dans un centre de soins de la faune sauvage. Son comportement était celui d'un animal domestique : elle venait spontanément vers l'être humain et restait près de lui ».

Ainsi, introduire un animal d'élevage dans un espace naturel, c'est bien évidemment le condamner à plus ou moins long terme. Cette pratique de la chasse, pour certains simulacres de retour vers la nature (Dalla Bernadina, 1996), n'est pas appropriation sensible 
d'un espace pour y sentir la vie sauvage où il s'agit de suivre des pistes animales, flairer, identifier des traces, se courber, faire silence, débusquer.

\section{Un territoire partagé et les « borderline »}

Sur un grand espace, les territoires humains et animaux s'entrecroisent parfois. La capacité de l'Homme à comprendre et à respecter les limites, à se faire respecter aussi, témoigne d'une possibilité de vivre dans un monde non artificiel où chacun peut avoir sa place. En Colombie Britannique, sur l'lle de Vancouver, quelques campings - très sommaires permettent aux adeptes des espaces peu fréquentés de vivre dans un environnement entre océan pacifique et forêt primaire. Les animaux tels que loups, ours noirs ou couguars évoluent aussi sur ce territoire. Dans cet espace partagé, la réglementation est stricte et vise à protéger tant les êtres humains que les animaux (caissons en métal pour la nourriture afin de ne pas attirer les animaux, se déplacer en faisant du bruit pour ne pas les surprendre, etc.). Tout animal observé est à signaler. Si la co-habitation se déroule apparemment sans tensions ni accidents, les possibilités de croiser un de ces mammifères restent faibles de toute façon. Mais les limites de la cohabitation sont nettes. Sont " hors-la-loi » ceux qui ne les respectent pas: pour les êtres humains, des gardes interviendront pour fautes de comportements (nourriture abandonnée sur une table par exemple), et comme personne ne tient nécessairement à se retrouver face à un ours, l'autodiscipline fonctionne. Les animaux ont eux "droit à l'erreur " parce qu'ils ne savent pas - ou presque - les conditions particulières exigées dans cet espace. Mais si la marge de manœuvre est plus grande quant au périmètre dans lequel ils peuvent évoluer, l'intervention à terme peut être radicale : un ours trop souvent à proximité humaine devient potentiellement dangereux et risque d'être abattu. Sur les panneaux de recommandations, il est rappelé que nos erreurs peuvent leur coûter la vie : "A fed bear is a dead bear ${ }^{7}$ nous invite à la responsabilité. Dans d'autres régions de l'île de Vancouver, les " choses " sont prises avec plus de légèreté et autorisent de fait une présence presque journalière de l'ours. II est alors important pour l'être humain de réaffirmer avec force sa présence et les limites de son territoire : l'ours n'a pas à être sur la poubelle blindée du camping ou près d'un logement tout en étant observé par les humains et sans être inquiété par eux. En effet, dans une telle situation de vis-à-vis, il prend ses aises et acquiert confiance. II faut agir pour le faire fuir, en lui fonçant dessus et en criant (et ça fonctionne!). L'ours sait fort bien qu'il n'a pas à être là, et repart tête basse et résigné en attendant un moment propice.

Dans ces espaces réduits où vivent êtres humains et animaux sauvages, les rencontres sont rares. Cependant, des règles de bonne conduite et de prudence sont fondamentales. A la périphérie d'un groupement de maisons, l'entrée d'un bois est habillée de cet écriteau temporaire improvisé sur un morceau de carton et qui invite à la vigilance : "Be careful. Mum and cubs in area ${ }^{8}$. Les animaux sont protégés dans le sens où l'on considère que la forêt est leur territoire. On ne criera pas vengeance si un homme paie de son imprudence ou

\footnotetext{
${ }^{7}$ Traduction personnelle : "Un ours nourri est un ours mort ».

${ }^{8}$ Traduction personnelle : «Soyez prudents. Une mère et ses petits dans le secteur ».
} 
de son intrépidité ${ }^{9}$. De la même façon, pour les ours trop présents en ville des sanctions peuvent être prises. Les "borderline " peuvent être abattus, mais ce n'est jamais un acte apprécié pour les gardes à qui incombe la tâche de tuer l'animal.

Comme évoqué précédemment, êtres humains et animaux savent poser les limites de leur territoire respectif, et comme pour tous les individus inter ou intra espèces, le " chacun chez soi » est à affirmer presque quotidiennement au risque d'un empiètement et d'une menace. Mais l'espèce humaine, colonisatrice, considère ne pas avoir de limites et pille constamment en presque toute impunité le territoire des autres êtres vivants. Pour Serres, l'appropriation de l'espace par l'être humain se manifeste par ailleurs sous forme de pollutions: " $L a$ croissance même de l'appropriation devient le PROPRE de l'Homme. Les animaux, certes, s'approprient leur gîte par leurs saletés, mais de manière physiologique et locale. Homo s'approprie le monde physique global par ses déchets durs (...) " (2008: 55). Et ces pollutions, indirectement, détruisent des habitats naturels et réduisent l'espace vital des autres espèces.

\section{Des cétacés et des Hommes : d'un espace de rencontre à un lieu de conflits}

Irlande, Bretagne, Caraïbes, Nouvelle-Zélande... qui n'a pas entendu parler de ces dauphins solitaires qui viennent à notre rencontre ? II en est ainsi de Jean-Floch venu côtoyer les ports bretons au début des années $2000^{10}$. La rencontre entre un humain et un animal sauvage peut-être surprenante, furtive, mais aussi s'installer dans la durée. Ce dauphin venait rechercher une sorte de compagnie et beaucoup de contacts physiques; il a fait de ce petit port finistérien son territoire de jeux et de repos. La première saison, le port était rempli d'observations et de moments de partage : nage et descente au fond de l'eau avec le dauphin, imitation de ses acrobaties, caresses, etc. Initialement, ce lieu était celui des pêcheurs qui ont donc accepté la présence du dauphin dont ils appréciaient initialement la compagnie, puis d'un nombre de plus en plus important de personnes jouant autour des embarcations. Mais progressivement des signes de détérioration dans la relation sont apparus jusqu'au conflit allant à l'atteinte de l'intégrité physique et psychique des protagonistes humains et animal.

L'échec de cette relation renvoie entre autres à la façon de concevoir et de comprendre l'animal sauvage qui justement échappe à l'Homme, à ce qu'il veut s'approprier. Le mythe de Flipper, la romantisation orchestrée dans le film Le Grand Bleu, le rêve de nager avec un dauphin a déclenché des comportements passionnés où l'envie du contact passait outre le respect de l'animal et des conseils avisés, comme si toucher le dauphin et s'accrocher à sa nageoire dorsale étaient un droit, un « à tout prix " que rien n'aurait pu empêcher. Sautant dans l'eau, surexcités, attendant parfois désespérément que le dauphin viennent à eux et au final, frustrations, tensions même entre les individus. Pierre, âgé d'une cinquantaine

\footnotetext{
${ }^{9}$ Tel ce photographe des grizzlis qui a été frappé mais non tué par l'un d'entre eux. Il avait déjà été verbalisé pour n'avoir pas respecté la distance minimale avec les grizzlys prévue par la loi canadienne. Pour plus d'informations, voir le site : http://blog.photojpl.com/attaque-par-un-grizzly/

${ }^{10}$ A la fois spectatrice et participante, j'ai passé de longues heures avec ce dauphin et observé mes homologues. Au final, un film et des témoignages qui facilitent une mise à distance face à une expérience riche de d'enseignements et de questionnements. Je n'en évoquerai ici que l'aspect difficile et complexe.
} 
d'années, naufragé sauvé par des dauphins, a été témoin privilégié de la rencontre entre des hommes et des femmes qui « se précipitaient » sur l'animal :

"Je disais aux gens qu'il fallait l'observer avant de faire quoi que ce soit, parce que s'ils n'étaient pas invités, ils allaient se faire sortir de l'eau plus vite qu'ils n'y étaient rentrés, "Mais ça, ça nous regarde " me répondaient-ils. Je savais exactement ce qui allait arriver : le dauphin a foncé sur eux en émettant des sons agressifs, les gens ont paniqué et il les a fait sortir! ».

Et puis le non-respect des règles élémentaires de sécurité, l'empiètement sur l'espace maritime des pêcheurs. La conséquence de tout ceci fut l'interdiction pour quiconque de se mettre dans l'eau en présence du dauphin devenu " une bête étrange..., monstrueuse..., vraiment là pour attaquer $\|^{11}$.

Les limites d'une telle relation sont complexes. II est question d'un territoire momentanément partagé où s'imbriquent des motivations différentes, que ce soit celles de l'animal ou des humains. Apparaissent alors des empiétements et des violations d'espaces à plusieurs niveaux : non respect de celui du dauphin, des pêcheurs, appropriation du port par le dauphin qui manipulait les nageurs à sa guise - il en faisait sortir certains de l'eau et en gardait d'autres de force - et au final des tensions multiples inter et intra-espèces (Héas, 2010). Ces échecs dans les relations humains/animaux ne peuvent que nous interroger sur nos propres comportements souvent inappropriés et nos représentations mystifiées, dans la version "Bambi " ou "Les dents de la mer", sur nos volontés de domination et d'appropriation du monde sauvage, nos représentations inadéquates pour traduire une intelligence de toute façon différente.

\section{De la distance respectée à la relation intime : " l'entre-deux animal »}

Plus loin que ces traques animales où l'Homme maintient une distance physique, cherche à observer sans être observé, vouloir rencontrer c'est croiser le regard, se sentir existant aux yeux de l'autre, c'est établir un lien avec des conséquences émotionnelles et psychiques d'une autre teneur, entre pensée rationnelle et intuition, contrôle et laisser-faire. Existent-ils des conditions pour la rencontre, tout particulièrement en termes d'espace ? Comment et sur quoi se constitue cet échange voire cet agir ensemble ? Quel est donc cet animal ? Ou plutôt qui est-il, qui est-elle, celui ou celle qui vient rencontrer l'être humain ? II est indéniable que cette relation est construite sur un attrait commun entre les deux espèces mais la motivation de cet autre reste insondable, au-delà d'une curiosité évidente : "Par le biais de leur attitude les orques cherchent aussi à communiquer avec nous, ils sont ouverts aux autres espèces, ils ont des interrogations ", souligne Marc. Les questionnements sont nombreux. Présentons ici ce que peut être une relation extraordinaire avec un animal sauvage, aux portes de la complicité et de l'intime, en montrant comment l'espace est investi par l'humain, comment il peut entrer en osmose avec lui puis progressivement en symbiose avec l'animal pour basculer ensemble dans cet « entre-deux animal ».

\footnotetext{
${ }^{11}$ Extrait d'un article de presse dans le journal Ouest-France du 11/08/06.
} 
En tout cas, espérée ou fortuite, peut-être risquée, souvent bouleversante, cette rencontre est bien décidée par l'animal qui accepte de se montrer et d'entrer en contact, et ce, tout particulièrement en mer. Imaginez cet instant : vous êtes seul(e) en maillot de bain avec un masque et tuba à la surface d'une mer chaude qui ouvre sous vos pieds plusieurs centaines de mètres de profondeur, du bleu sans fin et de plus en plus dense. Vous avez observé un souffle d'évent dans le lointain, la grande baleine est là-bas. Vous attendez que cet animal géant vienne se présenter à vous. Attendre. Espérer. Entre cœur qui bat la chamade et sentiment de confiance qui s'impose. Et puis le surgissement, à quelques mètres de vous...

\section{Les temps de la rencontre : être à l'écoute de soi, du milieu, de l'autre}

Comme un besoin impérieux, la rencontre, parfois renouvelée ${ }^{12}$ vient ponctuer l'existence de ces moments où l'individu est en quête d'une nourriture sensorielle, voire cognitive, entre sentiment d'une "intégration au cœur même de la nature ", émerveillement, excitation, et puis et surtout ce qui instaure ce lien privilégié entre soi et l'animal, " ce pont invisible entre deux univers sensoriels différents $»^{13}$. Ainsi, pour Marc qui rencontre annuellement les orques en Norvège: "Il n'était pas envisageable que je puisse arrêter là. Donc, tous les ans j'y retourne. Je me transforme dès que je pars là-haut, il faut que j'y aille ". Comme il le précise, les conditions mêmes du milieu vont être déterminantes :

"Le gros avantage de ce milieu rude, c'est qu'il est extrêmement sélectif. Tout le monde n'a pas forcément envie de vivre ça : une houle d'enfer, des journées où on ne voit pas le soleil, vivre le gros temps et les orques pas là, donc de la désespérance. On en a conscience, on sait où on est ".

A côté de ceux qui partent et osent l'aventure, sur le lieu même de l'expédition les attitudes divergent encore, une autre étape s'installe : "Tout le monde ne ressent pas le besoin de se mettre à l'eau. Certains préfèrent observer les orques depuis le bateau et ça leur suffit ». Etre immergé dans un milieu relativement inconnu, loin de tout repère, va instaurer une nouvelle relation à l'environnement. Notre éducation et notre culture conditionnent en partie notre sensorialité et notre mode perceptif puis interprétatif (Le Breton, 2007), " dès qu'on naît on est cloisonné dans plein de choses, au niveau du toucher par exemple ", précise JeanFrançois, vétérinaire de formation et cinéaste animalier. Or, pour accéder à ces expériences hors du commun, les enquêtés entrent dans une perception inhabituelle.

Les transformations que la relation avec l'animal sauvage engendrent et créent se situent déjà dans l'instantanéité de la rencontre, dans le face à face, où suivre son instinct et faire confiance sont d'importance. Les capacités d'adaptation au milieu, la façon de s'y immerger et d'y être sont essentielles. Bartabas parle de notre rôle dans notre " relation à l'autre, à la terre, au ciel et à notre animalité, de cet équilibre entre l'action et l'instant, notion fondamentale de l'être, qui nous échappe, à peine sorti de l'enfance, du mouvement dans son amplitude, sa rondeur ${ }^{14}$. A propos des orques, Marc décrit: "J'ai adopté immédiatement l'axe de nage du groupe et là, ils infléchissent et ils viennent. Ça s'enroule et ça vient ". L'observation comportementale et le ressenti aident à la prise de décision, à ce

\footnotetext{
${ }^{12}$ C'est le cas avec ces familles d'orques qui d'une année sur l'autre s'installent dans les mêmes régions, lieux de migration de bancs de poissons dont elles se nourrissent.

${ }^{13}$ Propos d'une jeune femme au sujet d'une femelle dauphin, in Matignon (2000 : 191).

${ }^{14}$ Cité in Matignon (2000 : 206).
} 
qui, non plus dans l'instantanéité, mais dans l'expérience des rencontres se construit progressivement et se précise : "Au fil de mes expériences, de mes erreurs, je me suis affiné dans mes trajectoires, mes positions de corps, ce qui me différencie un petit peu des débutants ». Un réel apprentissage se met ici en place, dicté par le désir d'être présent et signifiant pour l'autre, jusqu'à pouvoir interagir, un apprentissage qui dit l'intensité de l'engagement et l'envie de communiquer, d'échanger dans un même espace proche.

\section{S'imprégner du milieu et " se déshabiller »}

Dans ces contextes particuliers, détachés de certaines contraintes sociales, il semble que des hommes et femmes soient disposés ${ }^{15}$ à une écoute d'informations plus subtiles venant de l'environnement (Varvoglis, 1992). Progressivement s'installerait un état d'esprit, un état d'être contemplatif, accueillant - accueillir des paysages, des sons, des odeurs, des sensations. Les enquêtés témoignent d'une mise en phase avec le milieu, à son rythme pour "être sur la même longueur d'onde ". Une syntonie si aboutie qu'elle éveille ce sentiment d'appartenance, de "fusion ».

Il est également nécessaire de ne pas être parasité par un environnement, entourage compris, qui viendrait "brouiller " les échanges. Pour Marc : "Là-bas, on pense orque, on mange orque [soit des harengs], on dort orque, on nage orque... Chacun est un peu dans sa bulle ". II semble se profiler ici un état modifié de conscience tels que décrits par Varvoglis : l'être dans une situation loin du stress qui bloque une "conscience plus subtile " (1992) entre en interconnexion avec le monde environnant. "Lâcher le mental ", c'est se libérer de ses repères habituels, ses représentations, pour rester seul et au cœur de l'action. II s'agit d'un agencement spatial et humain, unique, dont l'issue est inconnue. Tout est ainsi question d'attitude, de disposition à et de prédisposition. Le rôle des sens est primordial et prépare le «terrain ».

Dans ses rencontres avec les orques, Marc précise :

"Ce qui rend ce moment très fort, c'est que ça s'est passé sous l'eau et que tu étais dans son univers. Donc tu es obligé de te déshabiller et de laisser beaucoup de choses sur le bateau pour pouvoir accéder à ça. Tout le monde n'en a pas la capacité. Il y en a qui passent à côté, même quand ils vont dans la flotte, ils restent terriblement humains $»$.

Se mettre ainsi à nu, psychiquement, est une des conditions de la rencontre, avec soi et avec l'animal. On se débarrasse de ce qui encombre, on se rend disponible pour être profondément dans l'instant, dans cet ici et maintenant. Cela nécessite de faire confiance, de dépasser des peurs. Pour exemple, Bartabas explique : " Les chevaux te voient l'intérieur. Tu peux te représenter à eux affublés d'un grand chapeau et d'un manteau, dès que tu auras fait trois mouvements, ils t'auront parfaitement identifié par ton aura, ton rythme intérieur,

\footnotetext{
${ }^{15}$ II y a des parcours de vie depuis l'enfance où peut se construire et siéger une relation à la nature préférentielle, et puis ces "traits de personnalités " à la recherche de sensations variées et nouvelles. La théorie de la recherche de sensations se fonde sur des concepts psychophysiologiques comme celui de niveau optimum de stimulation caractérisant chaque individu est défini comme " un trait de personnalité ». In André et al (2002).
} 
la manière dont tu te déplaces ${ }^{16}$. On ne peut de la même façon tricher face à un animal tel que l'orque.

\section{Du respect à la réciprocité}

Les " tours operators " qui vendent des prestations pour rencontrer les mammifères marins sont soumis à des chartes, conditions pour approcher le mammifère, obligations du navigant et des observateurs afin de ne pas nuire. Si ce code de bonne conduite institue un " savoir " faire, pénétrer physiquement dans l'univers de l'animal sauvage est exigeant en termes de respect et de capacité à appréhender l'autre, sinon l'animal prend la fuite. "Quand on dérange les orques, ils disparaissent sans rémission. Ils nous privent de leur présence, c'est principalement la punition qu'ils nous infligent ", souligne Marc. Respecter c'est reconnaître celui qui est en face en tant qu'être désirant ou non, accepter qu'il ait ses limites, un espace à ne pas franchir et si un contact par les corps est possible, il ne sera pas volé mais échangé. La liberté est un concept propre à l'être humain mais non applicable à lui seul. L'animal sauvage a le pouvoir de ne pas être "sous la main de l'Homme ", il est important de le reconnaître et de l'accepter comme entité, existence pleine et entière, dans son libre accomplissement.

"I'ai eu l'immense bonheur de vivre une expérience hors du commun avec une raie manta ${ }^{17}$. Un profond respect était là comme prélude, la connexion s'est faite dès la première plongée, dès le premier regard, mais avec cette distance maintenue entre nous, quand nous nagions dans l'œil l'une de l'autre, à la même vitesse, dans cette douceur extrême, entre fluidité des mouvements et harmonie. Je l'ai laissée venir, après plusieurs heures de mise en présence, jusqu'à cette ultime plongée où un long contact physique s'est installé ».

Avec les orques la situation est nécessairement plus précipitée, plus dans l'urgence, l'excitation. Parfois la rencontre se fait sur un intervalle de quelques secondes seulement, le temps d'un regard. Et puis les conditions du milieu, l'incertitude même de leur présence participent de cette atmosphère, de cette tension. Si apprivoisement il $\mathrm{y} a$, il se construit aussi d'années en années - ce sont les mêmes familles d'orques qui reviennent - comme pour Marc qui accomplit ce voyage depuis plus de dix ans :

"Maintenant, avec l'expérience, on a appris à décrypter dans leur comportement à partir de quand ils acceptent un peu la pression, l'approche non intrusive, à partir de quand ils nous acceptent et quand ça les dérange. Là, on recule et on attend ».

Cet "à partir de quand " définit une échelle de précision de l'ordre du sensible. C'est en observant par exemple des détails dans les mouvements du corps, les sons émis, les distances imposées mais aussi dans un sentiment subtil que l'on ressent le jeu de la rencontre, le possible ou non, l'accord ou la dysharmonie avec l'animal.

\footnotetext{
${ }^{16}$ Cité in Matignon (2000 : 207).

${ }^{17}$ Cette rencontre a eu lieu en 2001, en Mer de Cortez.
} 


\section{Des rencontres, des « entrecroisements » et au seuil regarder}

L'animal a cette capacité de nous mettre en mouvement au travers de champs de forces émotionnelles que nous partageons avec lui et qui induisent et façonnent nos comportements : étonnement, curiosité, excitation, plaisir, etc. Les communications non verbales inter espèces s'entourent d'une expérience des affects presque indicible et d'une nature délicate à objectiver pour ce qui concerne l'animal: l'expérience n'est pas reproductible et nous ne pouvons parler à sa place. Mais avons-nous seulement à la rendre intelligible pour la rendre légitime?

" Je souhaitais pouvoir être seule avec la raie manta, l'effleurer même du bout des doigts. J'ai décidé de quitter le groupe de plongeurs. Je n'osais pas imaginer le bonheur d'une autre rencontre et vivais dans un sentiment confus entre désir et espoir. J'ai finalement contourné le massif rocheux pour glisser doucement dans le bleu. Et puis elle est apparue devant moi. Avait-elle de la même façon quitté les autres raies pour me retrouver? ".

Si pour Collot « la communication entre inconscients nécessite un contexte affectif et un état de disponibilité mentale (...) une communication silencieuse d'une structure inconsciente à une autre " (2009: 148), je peux penser qu'au vu de l'agencement et du déroulement progressif de la journée, nous étions toutes deux engagées et tendues vers cette relation. Nous étions là, loin des autres, seules mais ensemble. Alors quand elle m'est passée audessus, m'offrant sa face inférieure, je n'ai eu qu'à poser mes mains, et elle nous a emmenées dans une des clairières sous-marines où elle s'est mise à tourner avec lenteur. Je suis restée suspendue sous ses ailes de longues minutes. II y a eu un accord complet de nos deux entités, une totale fluidité. Alors, que cette aventure soit extraordinaire et unique n'enlève rien à sa réalité : elle a eu lieu, et je peux donc maintenant ouvrir un espace d'interrogations. J'ai pu communiquer avec cet animal sauvage, partager avec lui un long moment qui n'est pas que l'instant furtif d'une rencontre et d'un regard. Nous avons évolué ensemble plusieurs heures jusqu'à cette douce complicité. Pour ce qui lui appartient, je peux seulement dire qu'elle est venue me chercher. Pour ce qui me concerne, cette relation participe de fait, au creux de et par-delà l'expérience, à l'évolution de ce qui me constitue comme sujet. Ces émotions, ces ressentis, tout ce qui m’a fait vibrer dans ma subjectivité, c'est cet autre animal qui l'a initié.

\section{« L'entre-deux animal ". Un espace de liberté pour les deux espèces}

Au sujet de ces rencontres entre l'Homme et l'animal, Bailly souligne avec justesse : "Ce qui est en jeu ici, ce n'est pas l'imitation, par les animaux, de processus mentaux humains, mais c'est une ressemblance étale dont les regards justement sont l'écho - un peu comme si en deçà des particularités développées par les espèces et les individus existaient une sorte de nappe phréatique du sensible, une sorte de réserve lointaine et indivise, incertaine, où chacun puiserait mais dont la plupart des hommes ont appris à se couper totalement, si totalement qu'ils n'imaginent même plus qu'elle puisse exister et ne la reconnaissent pas quand pourtant elle leur adresse des signes » $(2007,46)$.

Dans l'espace de cet entre-deux, être humain et animal ont juste à exister ensemble. Qu'elle soit de dix secondes ou de quelques minutes, cette zone acquiert une propriété toute particulière : elle se crée au sein de cette rencontre et de ce partage entre les deux espèces où, pour l'humain, se vit à la fois une attente et un "complètement là ", plein et subtil, 
presque naïf. II est même préférable que ce temps ne soit pas trop long car l'esprit aurait alors tôt fait de tergiverser sur ce qui n'est pas si important dans l'instant : qui est cet animal ? Trop court parce qu'il est un moment d'extase rare et précieux. II ne s'agit pas ici d'une expérience conforme à une conception du monde, telles les modalités ontologiques que sont l'animisme ou le naturalisme mais d'une expérience non prédictible, unique, profondément intime et d'une force incroyable, "(...) quelque chose de sacré... ", "(...) quelque chose qui dépasse notre entendement", confient respectivement Pierre et Marc. Vient ensuite le temps de l'étonnement devant ce croisement de vies, puis les premières questions qui surgissent et l'ébranlement, si on l'accepte. Marc témoigne :

"Cette expérience-là m'a enrichi énormément. Après, tu perçois les choses qui t'entourent avec un autre regard. Ces expériences ne modifient pas que ton échelle de valeurs, mais aussi ton fonctionnement ».

Ces hommes et ces femmes sont aux aguets dans leurs explorations en nature, dans la traversée des mondes animaux : ils sont dans le désir d'une rencontre, d'un quelque chose qui pourrait les surprendre. Il y a donc cet " être aux aguets » qui est tension vers, prélude à. Mais, si être animal, c'est être aux aguets dans le sens d'une tension quasi permanente d'écoute et de vigilance dans un monde de prédateurs et de proies, cet entre-deux de la rencontre offre aussi un répit existentiel pour ces deux espèces : pour l'être humain car il échappe dans un espace-temps aux contraintes et aux tensions d'une société de vivants hautement organisée et complexe qui attend que chacun réponde en permanence de sa position. Je ne parlerai pas au nom de l'animal qui reste sauvage et repart dans sa vie, auprès de ses congénères.

Cet " entre-deux animal », ce mouvement suspendu, est enfin espace de création, presque un art dans l'être lié, l'aboutissement d'une façon d'être au monde. Homme et Animal auront quitté leur territoire pour en découvrir et en partager un nouveau et, nourris de cette expérience, ils repartiront vers leur territoire d'origine qui sera alors enrichi et transformé. Les territoires se dessinent et se redessinent, indéfiniment. Et si ces territoires humains et animaux - qu'ils soient géographiques ou psychiques - se mélangent parfois, il n'y a pas de perte d'identité mais une expérience de l'altérité où percevoir et éprouver produisent du sens par-delà les affects et deviennent une force d'enracinement.

\section{Ce qui est en marge de... Des singularités et des potentialités}

Les faits sont en eux déjà " quelque chose ", ils font sens. Même si nous ne pouvons à partir de quelques rencontres, à caractère somme toute exceptionnel, avancer une réalité du monde animal, nous montrons déjà que les enquêtés, par leurs capacités réceptives et sensibles, perçoivent celui-ci en bousculant les connaissances. Pour exemple, tel animal rencontré se définit selon une nomenclature qui permet de le situer dans l'échelle du vivant: la raie manta, Manta birostris, de l'embranchement des vertébrés, poisson cartilagineux de la famille des Mobulidae, etc. Ainsi est reconnu à tel être vivant telle capacité motrice, perceptive... Ce qui est intéressant est le vécu, les comportements des protagonistes, ce qui s'est passé dans cet instant de la rencontre et qui de toute façon dépasse pour l'animal les considérations liées aux sciences naturelles. " Dis-moi comment tu 
conçois le composé humain, et je te dirai ta position sur les phénomènes paranormaux » nous dit Meinrad (1998: 7). II me semble que nous pouvons transférer cette remarque aux conceptions du monde animal.

Dans ces rencontres tellement surprenantes et inattendues, soudaines, dans ce surgissement, c'est un autre mode d' "être avec " qui s'impose. Les relations singulières entre un être humain et un animal sauvage poussent les limites de l'altérité, brouillent nos repères et bousculent nos représentations du monde vivant, mais pas seulement. Elles sont aussi vectrices de transformations pour celles et ceux qui les vivent (Ormiston, 2003) et soulèvent nombre d'interrogations quant aux potentialités humaines et animales. Chacun, en fonction de son parcours de vie, de son histoire, de la trame familiale, sociale dans laquelle il s'inscrit et évolue, vit profondément et intimement sa relation à la Nature, à l'Animal.

Parmi ces expériences en nature, des situations inhabituelles, atypiques se présentent et viennent semer le doute : la perturbation a en effet quelque chose à nous dire. Au sujet de la diversité en tant que réalité naturelle, Gould souligne: "Nous pensons une tendance comme le mouvement d'une entité dans une certaine direction, alors qu'elle peut être la conséquence secondaire d'un accroissement ou d'une diminution des variations au sein d'un système, de l'ouverture ou de la fermeture de l'éventail des possibles » (1997 : 49). II s'agit de considérer les variations du système entier et non de se focaliser systématiquement sur des mesures abstraites que reflète la tendance centrale. Tout ce qui sort de l'ordinaire, ce fameux contre-exemple, nous pousse à relativiser et à penser le multiple, l'imprévisible. L'hétérogénéité des vivants montre ses variations, ses mélanges et ses inattendus. A la notion de développement nous préférons celle de déploiement avec son amplitude et son ouverture. Certains se distinguent par une singularité, un quelque chose d'étonnamment différent, voire de résistant, et ceux-là même, indirectement, infléchissent un mouvement (Moscovici, 1996).

\section{Un espace défini par le désir. Des variables entre appropriation et acceptation, fermeture et ouverture}

Est-ce notre intention dans la relation qui dessine un éventuel espace, ses contours, ou estce la prédétermination d'un espace choisi en fonction de notre conception du monde qui définit le type de relation ? Dans le premier cas la marge de manœuvre est plus grande, tout espace reste possible et se construit, l'espace n'est pas prioritaire, il est habité en fonction de l'intentionnalité de l'animal et de l'être humain, du niveau de la relation, et du type d'échange. C'est l'exemple des explorations en nature motivées par le souhait d'une rencontre, souvent fortuite. $S^{\prime}$ il peut y avoir une déception, elle est largement compensée par ce que l'individu aura mis en œuvre et déployé pour tenter d'atteindre un objectif : déplacement, engagement physique, sensations corporelles, émotions ressenties en étant au cœur de la nature, attente, écoute, espoir, tout ce qui participe de l'avènement ou non de la rencontre. Soit une figure du concept de désir énoncé par Deleuze : "On désire un ensemble et non quelqu'un ou quelque chose (...) désirer c'est construire un 
agencement ${ }^{18}$. Ainsi, désirer rencontrer un animal sauvage c'est s'installer dans un contexte ouvert à tout changement, dans un ensemble d'éléments qui ne sont pas seulement l'animal, mais tout ce qui est agencé et s'agence à la mesure que se déroule le temps : les conditions du milieu, l'humain en évolution corporelle, sensible et psychique. Si la rencontre n'a pas lieu, l'homme ou la femme aura de toute façon apprécié le temps vécu : un paysage, des odeurs, des lumières, etc. II n'y aura pas de grande déception.

Parfois ces rencontres, lorsqu'elles sont renouvelées, comme dans l'exemple des dauphins solitaires, induisent des sentiments liés à la frustration ou à la déception, soit parce qu'il y a une sorte d'apprivoisement réciproque avec ses tensions, soit parce que l'être humain est dans un imaginaire, ou bien le contexte dans lequel se déroule la rencontre n'est pas appréhendé, il y a focalisation sur l'animal et lui seul, il n'y a pas d'agencement vécu. A-t-on seulement apprécié la nature tout autour ? Nos idéaux, nos projections sur le monde animal nous éloignent parfois de la réalité et quand nous pensons atteindre un moment de félicité dans une rencontre somme toute un peu atypique et magique, nous pouvons aussi nous retrouver face à un grand décalage entre relation fantasmée et vécu. "L'objet " de notre motivation se transforme alors en source de mécontentement, $d$ 'incompréhension, voire de colère. Nous pensions avoir droit à, comme un dû, et nous nous en sommes déçus ou privés. L'animal réagit, lui aussi.

Dans l'exemple du zoo ou du parc animalier aquatique, l'espace est immuable et défini une fois pour toute - à l'exception des transgressions aux conséquences souvent catastrophiques (une main passée à travers un grillage, un animal qui s'échappe). II est ici question d'une totale maîtrise de l'espace (Ost, 1995 ; Staszak, 1999) dans lequel est introduit un animal initialement sauvage et donc une maîtrise de la relation avec l'animal : on pourra l'observer à sa guise - à moins que l'animal soit cloîtré dans son refuge - une certaine proximité peutêtre rendue possible avec des cloisons en plexiglas par exemple, il sera photographié, voire touché, etc. Le visiteur paye pour une prestation et le gérant souhaite bien sûr qu'il ne soit pas déçu. L'espace du zoo est un espace fermé malgré les apparences d'ouvertures et de possibles mouvements tant pour les animaux que pour les humains qui y sont guidés, un espace conquis par avance où l'incertitude n'a pas sa place. Mais dans une société qui nous dit que tout est accessible à moindre coût financier, temporel, en termes d'engagement physique ou émotionnel, le monde sauvage nous renvoie à la non accessibilité, à la non immédiateté relationnelle, si toutefois celle-ci est possible. Patience, efforts sont alors conjoints à ces moments d'extase. Parce que le photographe amateur qui saisit des instants du brame des cerfs aura passé des heures à l'affût, parce que ce plongeur aura affronté l'eau glaciale pour peut-être vainement croiser le regard d'un orque, parce qu'aucune certitude ne peut nous assurer de ce que nous voulons jouir, alors il nous faut accepter la vie sauvage, la rencontre éphémère, la Nature, avec ses lois qui limitent nos champs d'actions et de succès.

\footnotetext{
${ }^{18}$ In Boutang (2004).
} 


\section{Conclusion}

\section{Intériorité et extériorité, et les nouveaux territoires}

Les rencontres et les relations avec l'Animal, tout particulièrement lorsqu'il s'agit d'un être sauvage encore peu imprégné par l'Homme, s'inscrivent dans un espace aux multiples dimensions et natures. A côté de l'espace géographique, du jardin à l'océan, se dessine parallèlement l'espace intime qui interfère avec celui-ci autour des notions du ressenti, de l'approche, de la distance de fuite jusqu'à la proximité. En effet, l'individu peut découvrir et s'imprégner d'un nouvel espace, le faire sien comme espace identifié de sécurité, le faire évoluer dans la rencontre en fonction de ces appréhensions ou du comportement de l'animal, craintif ou curieux, du retrait au rapprochement. Evoluer dans un espace de rencontre, c'est donc jouer plus ou moins consciemment avec un ensemble de facteurs qui appartiennent aux deux protagonistes tout en gardant moult incertitudes au sujet de l'animal.

"L'entre-deux animal » est lui l'aboutissement d'une intimité entre deux êtres, d'une " complicité ", d'où les contraintes extérieures, pour un temps, sont exclues. Cet entre-deux est la conjugaison d'un agencement et d'un désir partagé, et de fait il ne peut se définir qu'en référence à une situation et une seule. Chaque être humain et chaque animal crée ainsi de nouveaux territoires et de nouvelles modalités de relations.

Le sens de l'intériorité et de l'extériorité devient ainsi fondamental parce qu'il est potentiellement communication avec soi et avec l'autre, communion avec un milieu. Corps, intimité, espace, extériorité, et l'autre. Ce nouvel espace d'interactions sera garant de cette capacité à renverser les valeurs et à voir l'autre autrement, par une ouverture à une autre conscience, différente. C'est pourquoi nous privilégions dans le discours des informateurs l'émotionnel, les affects, la perception et la sensorialité dans le vécu corporel (André et al, 2002), l'imaginaire (Durand, 1992), l'inédit, tout ce qui participe de l'expérience humaine, tout ce qui crée du sens au sein et autour des réalités vécues. L'intuition (Gladwel, 2006), le sens du mouvant, apportent flexibilité à la pensée rationnelle. Elle est associée à une logique bien particulière car : " C'est une logique de l'aléatoire et de l'incertain dont nous avons dès lors besoin pour penser les systèmes ouverts et complexes "(Ost, 1995, 246). Entre rationalité et interprétation subjective, de nouvelles voies d'exploration sont à entreprendre. Pour une interprétation du monde animal, l'agencement des rencontres, nombre de questions sont à poser. Nous ne pouvons exposer ici notre méthodologie de la rencontre animale mais soulignons : "Quand nous étudions l'intelligence et la créativité des animaux, il est évidemment nécessaire qu'en tant que scientifiques nous soyons créatifs avec nos méthodes » (Bates \& Byrne, 2007) ${ }^{19}$.

La synergie des relations, leurs richesses et leurs complexités, nous donnent ainsi la possibilité de créer des agencements aux résonances multiples. Ce qui altère, crée un mouvement d'involution et d'évolution. L'expérience radicale de l'altérité qui bouscule nos acquis, interroge nos certitudes comme nos manques mais surtout la façon que nous avons d'habiter le monde et d'être changé par lui. Les conflits pour l'espace sont des problèmes

\footnotetext{
19 Traduction personnelle: "When studying the intelligence and creativity of animals, it is surely necessary that we as scientists are creative with our methods".
} 
inhérents à nombre d'espèces vivantes. A côté de la loi du plus fort et du " struggle for life " qui servent à bon compte nos dogmes socio-économiques, apparaissent les phénomènes de symbioses, d'entraides comme voies d'évolution. La question n'est pas de savoir quel monde sauvage nous voulons, ce serait encore faire preuve d'une volonté de domination et de maîtrise, mais si nous sommes capables et sur quels modes, de cohabiter avec des animaux sur lesquels nous n'aurons pas d'emprise. L'autre animal n'est plus ce qui est à bannir, détruire, ou à transformer à notre convenance, mais ce qui partage notre existence sur cette terre, à sa place, à un moment donné de l'évolution.

\section{Bibliographie}

André J. et al. (2002), La vie sensorielle - La clinique à l'épreuve des sens, Paris, PUF

Bériot L. (2012), Ces animaux qu'on assassine. Trafics, mafias, massacres, Cherche Midi.

Bailly J.-C. (2007), Le versant animal, Paris, Bayard.

Bates L. A., Byrne R. W. (2007), "Creative or created: Using anecdotes to investigate animal cognition", Methods (42), 12-21

Boutang Pierre-André (2004), L'abécédaire de Gilles Deleuze, Editions Montparnasse.

Cardonne F. (2004), Approcher l'animal en milieu naturel, Actes sud.

Céfaï D. (2003), L'Enquête de terrain en sciences sociales, Paris, La Découverte.

Chanvallon S., Héas S. (2011), "L'Homme et la Nature: en-quête/enquête sensible", Natures, Sciences, Sociétés, 19-4, pp. 355-364.

Chanvallon S. (2009), "Anthropologie des relations de l'Homme à la Nature: la Nature vécue entre peur destructrice et communion intime ». Thèse de sociologie, réalisée dans le cadre du LARES, membre du Laboratoire d'anthropologie et de sociologie [LAS], EA2241, Université Rennes 2.

Collot E. (2009), La conscience dans tous ses états: approches anthropologiques et psychiatriques, Masson, Paris

Cuche D. (2004), La Notion de culture dans les sciences sociales, Paris, La Découverte.

Dalla Bernadina S. (1996), L'Utopie de la Nature. Chasseurs, écologistes et touristes, Paris, Imago.

Durand G. (1992), Les Structures anthropologiques de l'imaginaire, Paris, Dunod.

Galinié H. (07/02/01), "Utiliser la notion de "distance critique " dans l'étude de relations socio-spatiales ", Les petits cahiers d'Anatole, 7.

Gladwell M. (2006), La Force de l'intuition, Paris, Robert Laffont.

Gould S. J. (1997), L'éventail du vivant, Paris, Seuil.

Gould S. J. (2001), Les Coquillages de Léonard : réflexions sur l'histoire naturelle, Paris, Seuil.

Héas S. (2010), Les discriminations sportives dans les sports contemporains ; entre inégalités, médisances et exclusions, Nancy, PUN, collection Epistémologie du corps.

Héas S. (2010), Les Virtuoses du corps : enquête auprès d'êtres exceptionnels, Paris, Max Milo.

Hennel-Brzozowska A. (2008), " La communication non-verbale et para verbale. Perspectives d'un psychologue », Synergies Pologne, 5, pp. 21-30.

Lavigne C. (2007), « À qui appartient l'objet de recherche ? Penser l'implication du chercheur dans son objet : le handicap (surdité) ", Nouvelle revue de psychosociologie, 2, 4, 25-39.

Le Breton D. (2007), Anthropologie du corps et modernité, Paris, Boché.

Le Grand J.-L. (2006), "Implexité : implications et complexité » (http://www.barbierrd.nom.fr/JLLeGrandlmplexite.html). 
Lorenz K. (1985), Il parlait avec les mammifères, les oiseaux et les poissons. Tous les chiens, tous les chats, Flammarion.

Matignon Karine Lou (2000), Sans les animaux, le monde ne serait pas humain, Paris, Albin Michel.

Maxwell J.A. (1999), La Modélisation de la recherche qualitative : une approche interactive, Fribourg, Éditions universitaires de Fribourg.

Meinrad E. (1998), La rationalité d'un discours africain sur les phénomènes paranormaux, Paris, L'Harmattan.

Moscovici S. (1996), Psychologie des minorités actives, PUF, collection Quadrige.

Ormiston A. (2003), " Hegel on negotiating tanscendence ", Ethnologies, 25, 1, 55-86.

Ost F. (1995), La nature hors la loi - l'écologie à l'épreuve du droit, Paris, La Découverte.

Picq P. (2005), "De l'hominisation au développement durable : d'un paradigme à l'autre ", communiqué de presse du 25.01.05 (http://WWW.forum-events.com/debats/synthesepascal-picq-90641.html).

Radin D. (2000), La conscience invisible - le paranormal à l'épreuve de la science, Paris, Presses du Châtelet.

Serres M. (2008), Le Mal propre. Polluer pour s'approprier ?, Paris, Le Pommier.

Staszak J.-F. (1999), Actes du Colloque " Géographie et Nature », FIG (Festival International de Géographie), Saint-Die-des-Vosges.

Varvoglis M. (avec la collaboration de Christine Hardy) (1992), La rationalité de l'irrationnel. Une introduction à la parapsychologie scientifique, Paris, InterEditions. 